\title{
A Prognostic Model Incorporating Red Cell Distribution Width to Platelet Ratio for Patients with Traumatic Brain Injury
}

\author{
Ruoran Wang' \\ Min $\mathrm{He}^{2}$ \\ Jing Zhang' \\ Shaobo Wang ${ }^{3}$ \\ Jianguo Xu $\mathbb{D}^{1}$ \\ 'Department of Neurosurgery, West \\ China Hospital, Sichuan University, \\ Chengdu, Sichuan Province, People's \\ Republic of China; ${ }^{2}$ Department of \\ Critical Care Medicine, West China \\ Hospital, Sichuan University, Chengdu, \\ Sichuan Province, People's Republic of \\ China; ${ }^{3}$ Department of Infectious \\ Diseases, Xi'an Hospital of Traditional \\ Chinese Medicine, Xi'an, Shannxi \\ Province, People's Republic of China
}

Correspondence: Jianguo $\mathrm{Xu}$

Department of Neurosurgery, West China Hospital, Sichuan University,

No. 37, Guoxue Alley, Chengdu, 61004I, People's Republic of China

Email xujg@scu.edu.cn

Shaobo Wang

Department of Infectious Diseases, Xi'an Hospital of Traditional Chinese Medicine, No. 69, Fengcheng 8th Road, Xi'an, 710016, People's Republic of China Email 1307799083@qq.com
Background: As an inflammation-based marker, red cell distribution width to platelet ratio (RPR) has been verified to be associated with disease severity and outcome in many clinical settings. We designed this study to evaluate the prognostic value of RPR in patients with traumatic brain injury (TBI).

Methods: A total of 420 patients admitted with TBI were included in this study. Laboratory and clinical data were collected from an electronic medical record system. Univariate and multivariate logistic regression analyses were sequentially performed to discover risk factors of in-hospital mortality. Receiver operating characteristic (ROC) curves were drawn to confirm the predictive value of different markers including RPR in training set and testing set.

Results: Non-survivors had higher level of RPR than survivors $(\mathrm{P}<0.001)$. Logistic regression analysis showed that RPR was significantly associated with mortality even after adjusting for confounding factors $(\mathrm{P}<0.001)$. The area under the ROC curve (AUC) value of Glasgow Coma Scale (GCS) for predicting mortality was 0.761 and 0775 in training set and testing set, respectively. And the constructed predictive model incorporating RPR had the highest AUC value of 0.858 and 0.884 in training set and testing set.

Conclusion: RPR is significantly associated with mortality in TBI patients. Utilizing RPR to construct a predictive model is valuable to evaluate prognosis of TBI patients.

Keywords: red cell distribution to platelet ratio, traumatic brain injury, prognosis, marker

\section{Introduction}

Traumatic brain injury (TBI) is a serious public health issue which brings a heavy burden to social economy and individual families. ${ }^{1}$ It was concluded that approximately sixty-nine million people would suffer TBI each year in the world. ${ }^{2}$ Children and young adults account for the majority of victims suffering TBI, and TBI has become the leading cause of long-term disability among them. ${ }^{3-5}$ TBI involves a series of pathophysiological processes including initial hit, neuroinflammation, inadequate cerebral perfusion and metabolism, excessive oxidative stress and excitotoxicity which are commonly associated with poor outcome in TBI patients. ${ }^{6}$ To make appropriate treatment decisions, some prognostic models have been developed and validated to predict outcome of TBI patients, such as IMPACT and CRASH models. ${ }^{7-9}$ And the trend of developing new prognostic models in TBI patients would not be terminated in future.

Complete blood count, comprising white blood cells, erythrocytes and platelets, is an accessible and routine laboratory test in clinical work. The measuring and 
morphological parameters of blood cells have been verified as valuable in evaluating severity and predicting outcome in various clinical settings. For instance, neutrophil to lymphocyte ratio (NLR), platelet to lymphocyte ratio (PLR), and red cell distribution width (RDW) have been generally acknowledged as inflammation markers in many diseases including cancer, cardiovascular diseases, and autoimmune diseases. ${ }^{10-13}$ In addition, the RDW to platelet ratio (RPR) was recently proposed as a novel inflammation marker and verified as performing well in predicting outcome in some diseases including hepatic fibrosis, acute pancreatitis, myocardial infarction, burn injury, and colorectal cancer. ${ }^{14-18}$

RDW is a morphological indicator of volume variability of erythrocyte which has been generally utilized to distinguish types of anemia. However, the characteristic of RDW reflecting inflammation status has received much attention in recent years. Increased level of circulating inflammatory cytokines including tumor necrosis factor- $\alpha$ (TNF- $\alpha$ ), interleukin-1 $\beta$ (IL-1 $\beta$ ), and interleukin-6 (IL-6) could inhibit erythropoietin (EPO)-induced erythrocyte maturation and accelerate the release of larger reticulocytes into blood circulation which in turn increases the RDW value. ${ }^{19-21}$

The adherence of platelets to damaged vessels and release of platelet granules are crucial to the formation of blood clots. Moreover, platelets also play an important role in local inflammation. It can activate some types of white blood cells including granulocytes, lymphocytes, and monocytes, and promote them to release inflammatory cytokines. ${ }^{22}$ Markers combining these cells including platelet to neutrophil ratio (PNR) and platelet to lymphocyte ratio (PLR) have been confirmed to be independently associated with prognosis in some intracranial diseases. $^{23-25}$

Previous research studied the relationship between single RDW or platelet value and mortality in TBI patients. ${ }^{26-28}$ And one study explored the prognostic value of RPR on TBI patients four days after admission, in the United States. ${ }^{29}$ However, there were several limitations in this study, including lacking records of intracranial injury types and Injury Severity Score (ISS). Therefore, we designed this study to confirm the correlation between RPR on admission and mortality after adjusting for potential confounders, and verify the prognostic value of RPR on admission of TBI patients hospitalized in a Chinese medical center.

\section{Materials and Methods}

\section{Study Population}

Patients diagnosed with TBI and hospitalized in West China hospital between January 2015 and June 2019 were included in this study. There were several exclusion criteria: 1) patients admitted to hospital 6 hours after head injury; 2) patients transferred from other hospitals; 3) patients with history of surgery or infection within 1 month before injury; 4) patients "complicated" by other central nervous system diseases, cancer, immune diseases, and severe renal or hepatic disfunction; 5) patients whose records lacked included variables. Finally, 420 patients were included in this observational study. This study was approved by the Ethics Committee of the West China hospital, Sichuan University, and was conducted in accordance with the relevant guidelines and regulations. Written informed consent about participation in observational scientific research of each patient was routinely obtained during their hospitalizations based on our hospital's regulations.

\section{Data Collection}

Clinical information including vital signs and Glasgow Coma Scale (GCS) on admission was collected from electronic medical record system of our hospital. Blood samples were routinely collected for laboratory analysis once patients were admitted to our hospital. Therefore, we selected laboratory tests on the first day of admission as variables and calculated the ratio of RDW and platelets. Coagulopathy of trauma was diagnosed based on any of the following criteria: 1) the international normalized ratio $>1.6 ; 2$ ) activated partial thromboplastin time $>60$ seconds. Intracranial injury types including epidural hematoma, subdural hematoma, subarachnoid hemorrhage, intraventricular hemorrhage and diffuse axonal injury were recorded and confirmed based on signs of radiological scans. The outcome of this study was in-hospital mortality.

\section{Statistical Analysis}

Normally distributed variables were shown as mean \pm standard deviation whereas non-normally distributed variables were presented as median (interquartile range). And categorical variables were presented in the form of numbers (percentage). We performed Kolmogorov-Smirnov test to verify the normality of variables. Independent Student's $t$-test and Mann-Whitney $U$ test were performed respectively to compare the difference between normally distributed variables 
and non-normally distributed variables. In addition, $\chi^{2}$ test was utilized to analyze the difference of categorical variables. Univariate and multivariate logistic regression with forward stepwise method were performed to find risk factors associated with in-hospital mortality. Then, we divided our original cohort into training set and testing set with ratio of $7: 3$. Independent risk factors were combined to construct prognostic model by logistic regression using training set. Then, receiver operating characteristic (ROC) curves and decision curve analysis (DCA) were drawn to evaluate the

Table I Baseline Characteristics of Survivors and Non-Survivors

\begin{tabular}{|c|c|c|c|c|}
\hline Characteristics & Total $(\mathrm{N}=420)$ & Survivors ( $N=2|4,5| .8 \%)$ & Non-Survivors (206, 48.2\%) & $\mathbf{P}$ \\
\hline Age (years) & $43(25-57)$ & $43(23-58)$ & $43(27-55)$ & 0.474 \\
\hline Male & $321(76.4 \%)$ & $167(78.0 \%)$ & I 54 (74.8\%) & 0.429 \\
\hline \multicolumn{5}{|l|}{ Injury mechanism } \\
\hline Traffic accident & $262(62.4 \%)$ & $|2|(56.5 \%)$ & $14 \mid(68.4 \%)$ & 0.012 \\
\hline Failing injury & $84(20.0 \%)$ & $50(23.4 \%)$ & $34(16.5 \%)$ & 0.078 \\
\hline Stumble & $46(11.0 \%)$ & $23(10.7 \%)$ & $23(11.2 \%)$ & 0.891 \\
\hline Others & $28(6.7 \%)$ & $20(9.3 \%)$ & 8 (3.9\%) & 0.023 \\
\hline Systolic blood pressure $(\mathrm{mmHg})$ & $120(106-138)$ & $121(108-139)$ & $120(102-138)$ & 0.157 \\
\hline Diastolic blood pressure $(\mathrm{mmHg})$ & $72 \pm 17$ & $74 \pm 14$ & $71 \pm 19$ & 0.160 \\
\hline Anisocoria & II 6 (27.6\%) & $67(31.3 \%)$ & $49(23.8 \%)$ & 0.084 \\
\hline GCS & $7(5-8)$ & $8(6-12)$ & $5(4-7)$ & $<0.001$ \\
\hline ISS & $25(16-25)$ & $16(9-25)$ & $25(24-25)$ & $<0.001$ \\
\hline \multicolumn{5}{|l|}{ Laboratory tests } \\
\hline White blood cell $\left(10^{9} / \mathrm{L}\right)$ & $14.87(10.69-19.11)$ & $13.82(9.97-17.94)$ & $15.57(\mid 1.53-20.45)$ & 0.012 \\
\hline Platelet $\left(10^{9} / \mathrm{L}\right)$ & $104(67-165)$ & $139(90-204)$ & $80(49-125)$ & $<0.001$ \\
\hline RDW (\%) & $14.2(13.3-15.3)$ & $13.6(|3.0-| 4.7)$ & $14.8(14.1-15.9)$ & $<0.001$ \\
\hline Hemoglobin $(g / L)$ & $90(76-110)$ & $98(82-116)$ & $83(72-99)$ & $<0.001$ \\
\hline Glucose $(\mathrm{mmol} / \mathrm{L})$ & $9.60(7.04-13.20)$ & $7.89(6.28-10.19)$ & $12.36(9.09-15.74)$ & $<0.001$ \\
\hline Chloride $(\mathrm{mmol} / \mathrm{L})$ & III.2 (I05.7-II8.8) & $107.4(|03.4-1| 11.5)$ & $117.9(110.9-126.7)$ & $<0.001$ \\
\hline RDW to platelet ratio & $0.14(0.08-0.22)$ & $0.10(0.07-0.16)$ & $0.19(0.12-0.33)$ & $<0.001$ \\
\hline \multicolumn{5}{|l|}{ Radiological signs } \\
\hline Epidural hematoma & $43(10.2 \%)$ & $23(10.7 \%)$ & $20(9.7 \%)$ & 0.725 \\
\hline Subdural hematoma & $100(23.8 \%)$ & 31 (14.5\%) & $69(33.5 \%)$ & $<0.001$ \\
\hline Subarachnoid hemorrhage & 229 (54.5\%) & 101 (47.2\%) & $128(62.1 \%)$ & 0.002 \\
\hline Intraventricular hemorrhage & $22(5.2 \%)$ & $8(3.7 \%)$ & $14(6.8 \%)$ & 0.158 \\
\hline Diffuse axonal injury & $103(24.5 \%)$ & $4 \mathrm{I}(19.2 \%)$ & $62(30.1 \%)$ & 0.009 \\
\hline Coagulopathy & $152(36.2 \%)$ & $38(17.8 \%)$ & II4 (55.3\%) & $<0.001$ \\
\hline Length of ICU stay (day) & $3(1-17)$ & $13(0-26)$ & $2(I-5)$ & $<0.001$ \\
\hline Length of hospital stay (days) & $12(5-28)$ & $25(13-42)$ & $5(3-10)$ & $<0.001$ \\
\hline
\end{tabular}

Abbreviations: GCS, Glasgow Coma Scale; RDW, red cell distribution width. 
predictive value of different markers and the prognostic model in training set and testing set. $Z$ test was performed to compare the predictive value of these markers and the model. And Net Reclassification Index (NRI), Integrated Discrimination Improvement (IDI) were also used to prove the incremental prognostic value of constructed models compared with single GCS. A P value $<0.05$ was considered to be statistically significant. SPSS 22.0 Windows software (SPSS, Inc, Chicago, IL) was used for all statistical analysis.

\section{Results}

\section{Baseline Characteristics of Included Patients}

A total of 420 patients were included in this study, among whom 214 (51.8\%) were survivors and 206 (48.2\%) were non-survivors (Table 1). The average age of total patients was 43 (25-57) years. The gender ratio and age did not significantly differ between survivors and non-survivors. The initial GCS score of non-survivors was obviously lower than survivors ( 5 vs $8, \mathrm{P}<0.001)$ while ISS was higher in non-survivors (25 vs 16, $\mathrm{p}<0.001)$. Laboratory tests showed that non-survivors had lower levels of platelets $(80$ vs $139, \mathrm{p}<0.001$ ) and hemoglobin ( 83 vs 98, $<<0.001)$. And the levels of white blood cells ( 15.57 vs $13.82, p=0.012)$, RDW (14.8 vs 13.6, p<0.001), glucose (12.36 vs 7.89, $\mathrm{p}<0.001)$, chloride $(117.9$ vs $107.4, \mathrm{p}<0.001)$ and $R P R$ $(0.19$ vs $0.10, p<0.001)$ were significantly higher in nonsurvivors. Results of radiological tests showed nonsurvivors had higher incidence of subdural hematoma (33.5\% vs $14.5 \%, \mathrm{P}<0.001)$, subarachnoid hemorrhage $(62.1 \%$ vs $47.2 \%, \mathrm{P}=0.002)$ and diffuse axonal injury (30.1\% vs $19.2 \%, \mathrm{P}=0.009)$. Additionally, coagulopathy occurred more frequently in non-survivors $(55.3 \%$ vs $17.8 \%, \mathrm{P}<0.001)$. Non-survivors had shorter length of

Table 2 Univariate and Multivariate Logistic Regression Analysis of Risk Factors for Mortality

\begin{tabular}{|c|c|c|c|c|c|c|}
\hline \multirow[t]{2}{*}{ Characteristics } & \multicolumn{3}{|c|}{ Univariate Analysis } & \multicolumn{3}{|c|}{ Multivariate Analysis } \\
\hline & OR & $95 \% \mathrm{Cl}$ & $\mathbf{P}$ & OR & $95 \% \mathrm{Cl}$ & $\mathbf{P}$ \\
\hline Age & 1.005 & $0.995-1.014$ & 0.340 & & & \\
\hline Male & 0.833 & $0.531-1.309$ & 0.429 & & & \\
\hline Systolic blood pressure & 1.000 & $0.997-1.004$ & $0.84 I$ & & & \\
\hline Diastolic blood pressure & 0.990 & $0.978-1.001$ & 0.079 & & & \\
\hline Anisocoria & 0.685 & $0.445-1.054$ & 0.086 & & & \\
\hline GCS & 0.665 & $0.606-0.729$ & $<0.001$ & 0.707 & $0.635-0.788$ & $<0.001$ \\
\hline ISS & I.107 & $1.079-1.135$ & $<0.001$ & & & \\
\hline White blood cell & 1.038 & $1.008-1.069$ & 0.013 & & & \\
\hline Hemoglobin & 0.975 & $0.967-0.984$ & $<0.001$ & & & \\
\hline Glucose & 1.282 & $|.207-1.36|$ & $<0.001$ & 1.167 & $1.095-1.245$ & 0.023 \\
\hline Chloride & 1.108 & $1.080-1.137$ & $<0.001$ & & & \\
\hline RDW to platelet ratio & 391.688 & $54.859-2796.635$ & $<0.001$ & 6.276 & $1.295-30.42$ & $<0.001$ \\
\hline Coagulopathy & 5.739 & $3.676-8.959$ & $<0.001$ & 3.050 & $1.767-5.264$ & $<0.001$ \\
\hline Epidural hematoma & 0.893 & $0.474-1.68 \mid$ & 0.726 & & & \\
\hline Subdural hematoma & 2.973 & I.843-4.796 & $<0.001$ & 2.477 & $1.369-4.482$ & 0.003 \\
\hline Subarachnoid hemorrhage & 1.836 & $1.244-2.709$ & 0.002 & & & \\
\hline Intraventricular hemorrhage & 1.878 & $0.77 \mid-4.575$ & 0.166 & & & \\
\hline Diffuse axonal injury & 1.817 & $1.156-2.856$ & 0.010 & & & \\
\hline
\end{tabular}

Note: $P$ value in bold font in multivariate analysis indicates $P<0.05$.

Abbreviations: OR, odds ratio; $\mathrm{Cl}$, confidence interval; GCS, Glasgow Coma Scale; RDW, red cell distribution width. 
intensive care unit (ICU) stay and length of hospital stay ( 2 vs $13, \mathrm{P}<0.001 ; 5$ vs $25, \mathrm{P}<0.001)$.

\section{Univariate and Multivariate Logistic Regression Analysis of Risk Factors for Mortality}

In univariate analysis, we found that GCS, ISS, white blood cells, hemoglobin, glucose, chloride, RDW to platelet ratio, coagulopathy, epidural hematoma, subdural hematoma, subarachnoid hemorrhage, intraventricular hemorrhage and diffuse axonal injury were all associated with mortality (Table 2). However, after adjusting for confounders in multivariate analysis, only five factors including $\mathrm{GCS}(0 \mathrm{R}=0.707$,
$\mathrm{P}<0.001)$, glucose $(0 \mathrm{R}=1.167, \mathrm{P}=0.023), \mathrm{RPR}(0 \mathrm{R}=6.276$, $\mathrm{P}<0.001)$, coagulopathy $(0 \mathrm{R}=3.050, \mathrm{P}<0.001)$, subdural hematoma $(0 \mathrm{R}=2.477, \mathrm{P}=0.003)$ were significantly associated with mortality.

\section{Predictive Value of RPR and the Constructed Model}

We constructed the predictive model incorporating risk factors which were statistically significant in multivariate logistic regression analysis using patients derived from training set. ROC curves of single RDW or platelets, RPR and the constructed predictive model were drawn (Figure 1). The area under the ROC curve (AUC) value

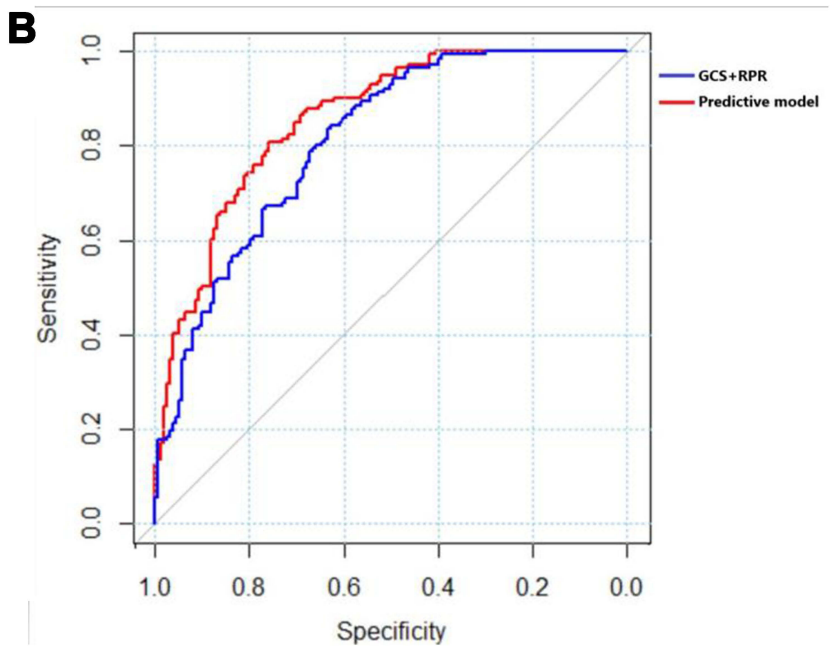

D

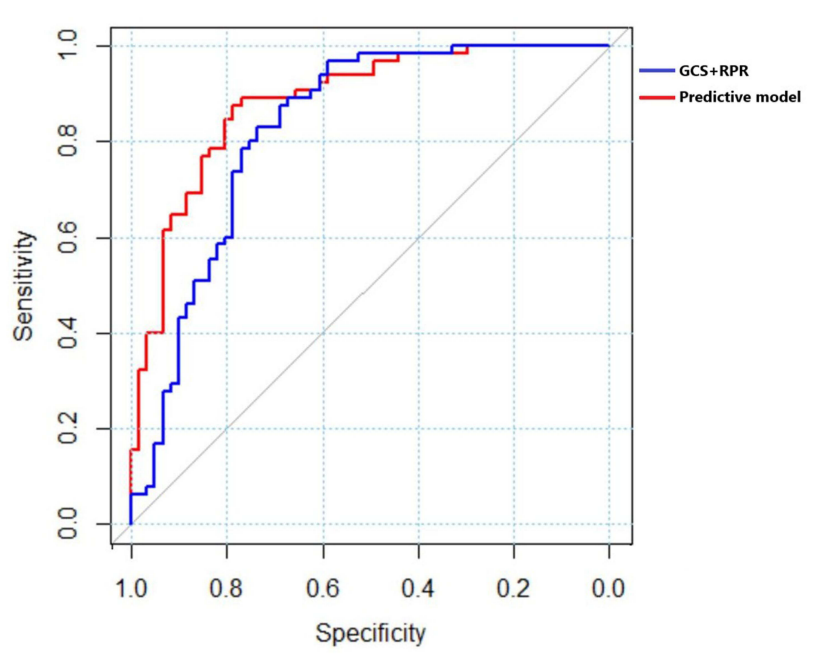

Figure I (A) ROC curve of single predictive markers in training cohort; (B) ROC curve of constructed prognostic models in training cohort; (C) ROC curve of single predictive markers in testing cohort; (D) ROC curve of constructed prognostic models in testing cohort. 
Table 3 Prognostic Value of Different Biomarkers and the Predictive Model in Testing Dataset and Testing Dataset

\begin{tabular}{|l|c|c|c|c|c|c|c|c|}
\hline \multirow{2}{*}{ Variables } & \multicolumn{4}{|c|}{ Training Dataset } & \multicolumn{4}{c|}{ Testing Dataset } \\
\cline { 2 - 9 } & AUC & $95 \%$ CI & Sensitivity & Specificity & AUC & $95 \%$ CI & Sensitivity & Specificity \\
\hline GCS & 0.761 & $0.708-0.815$ & 0.809 & 0.608 & 0.775 & $0.688-0.861$ & 0.908 & 0.639 \\
\hline RDW & 0.726 & $0.668-0.784$ & 0.745 & 0.66 & 0.735 & $0.645-0.824$ & 0.892 & $0.54 I$ \\
\hline Platelet & 0.738 & $0.681-0.794$ & 0.681 & 0.706 & 0.713 & $0.623-0.804$ & 0.462 & 0.902 \\
\hline RPR & 0.759 & $0.704-0.813$ & 0.660 & 0.745 & 0.733 & $0.645-0.822$ & 0.523 & 0.885 \\
\hline GCS + RPR & 0.810 & $0.762-0.858$ & 0.844 & 0.627 & 0.828 & $0.762-0.858$ & 0.831 & 0.738 \\
\hline Predictive model & 0.858 & $0.816-0.899$ & 0.809 & 0.758 & 0.884 & $0.826-0.943$ & 0.877 & 0.787 \\
\hline
\end{tabular}

Note: The predictive model was composed of five factors including GCS, glucose, RPR, coagulopathy and subdural hematoma.

Abbreviations: AUC, area under the receiver operating characteristics curve; Cl, confidence interval; GCS, Glasgow Coma Scale; RDW, red cell distribution width; RPR, red cell distribution width to platelet ratio.

Table 4 Improved Prognostic Value of Constructed Models Compared with Single GCS Demonstrated by NRI and IDI

\begin{tabular}{|l|l|l|l|l|l|l|l|l|}
\hline & \multicolumn{3}{|c|}{ Training Dataset } & \multicolumn{4}{c|}{ Testing Dataset } \\
\cline { 2 - 9 } & NRI & P & IDI & P & NRI & P & IDI & P \\
\hline GCS + RPR & 0.06720 & 0.081 & 0.0645 & $<0.001$ & 0.1538 & 0.015 & 0.0655 & 0.020 \\
\hline Predictive model & 0.2023 & $<0.001$ & 0.1578 & $<0.001$ & 0.2204 & 0.010 & 0.1902 & $<0.010$ \\
\hline
\end{tabular}

Abbreviations: NRI, Net Reclassification Index; IDI, Integrated Discrimination Improvement.

of single GCS was 0.761 and 0.775 in training and testing set, respectively (Table 3). And AUC value of RPR was 0.759 and 0.733 in training and testing set, respectively. Furthermore, the AUC value of GCS combined with RPR was 0.810 and 0.828 in two sets which was higher than single GCS $(\mathrm{Z}=1.3293, \mathrm{P}>0.05 ; \mathrm{Z}=1.1405, \mathrm{P}>0.05)$ and RPR $(Z=1.3835, P>0.05 ; Z=1.9825, P<0.05)$. Finally, the constructed five factor predictive model had the highest AUC value of 0.858 and 0.884 in training and testing set. The NRI and IDI indicated the constructed predictive model had incremental prognostic value than single GCS (Table 4). Decision curve analysis of the constructed predictive model in training set and testing set was presented in Figure 2.

\section{Discussion}

Evaluating trends of disease progression as soon as possible is beneficial for physicians to make appropriate treatment strategies to prevent damage of secondary brain injury in TBI patients. The GCS score, widely used as a convenient tool to evaluate severity, however, has some limitations such as unavailability in intubated patients. Recently, developing novel prognostic models of TBI has attracted much attention from researchers. In this study, we found that RPR was an accessible index to predict the prognosis of TBI patients. Moreover, utilizing RPR to construct a multi-factor prognostic model could increase predictive value.

Compared with survivors, non-survivors had higher level of RPR which generally meant higher RDW and lower platelet count together. A previous study has indicated that RDW might be a good predictor of 6-month neurologic outcome, but a poor predictor of mortality in TBI patients. ${ }^{26}$ The mechanism between higher RDW value and poor outcome has not been fully understood. There are some reasonable explanations for this association. Firstly, elevated RDW may indicate the existence of systemic inflammation. A study found that RDW level was associated with the level of common inflammatory markers such as high-sensitivity C-reactive protein (CRP) and erythrocyte sedimentation rate. ${ }^{30}$ The rise of inflammatory cytokines including TNF- $\alpha$, IL-1 $\beta$, and IL- 6 can inhibit erythropoietin (EPO)-induced erythrocyte maturation and then accelerate the release of juvenile reticulocytes into peripheral circulation which leads to the increased heterogeneity of erythrocyte volume. ${ }^{19-21}$ TBI can induce the expression of various inflammatory factors including CRP, TNF- $\alpha$, IL-1 $\beta$, and IL-6, which can aggravate cerebral 

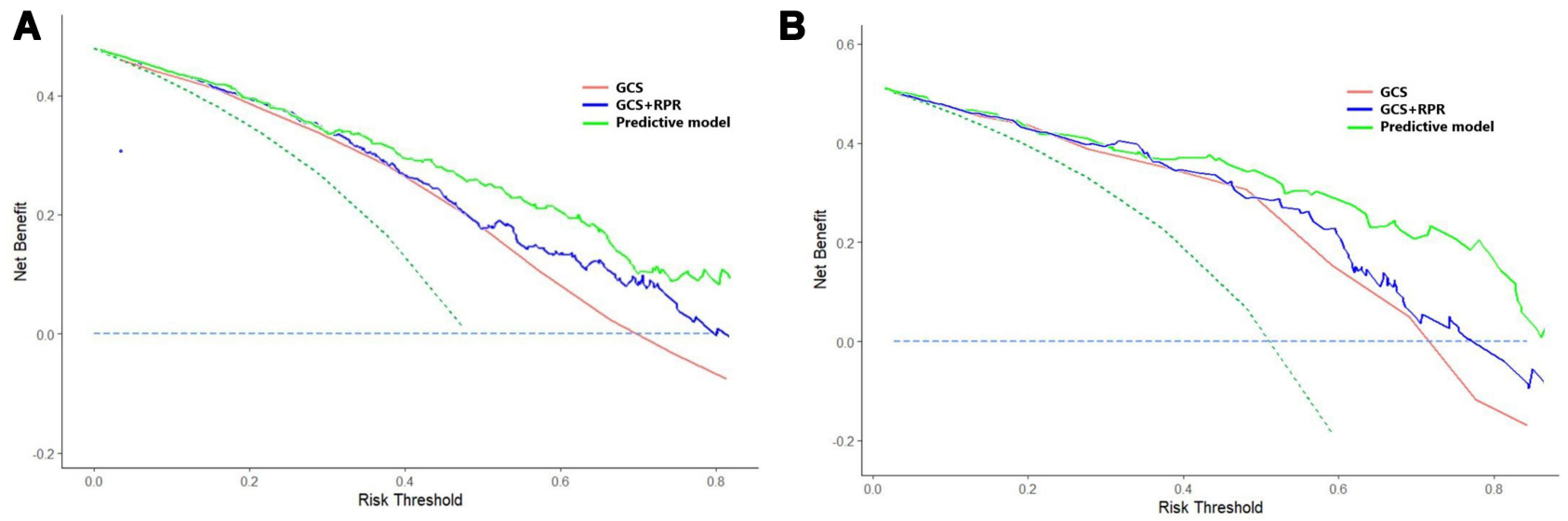

Figure 2 (A) Decision curve analysis of constructed models in training cohort; (B) decision curve analysis of constructed models in testing cohort.

injury and systemic organ damage. ${ }^{31}$ In addition, the relationship between systemic inflammatory response syndrome and outcome of TBI patients has been confirmed. ${ }^{32}$ Therefore, the relationship between increased RDW and poor outcome of TBI patients may be mediated by systemic inflammation. Secondly, oxidative stress can also inhibit erythropoiesis, and damage the deformability of erythrocyte membrane. As a result, the erythrocyte survival will be shortened with an elevated RDW. ${ }^{33}$ The deleterious effect of oxidative stress on neuronal dysfunction and death has been illustrated in TBI patients. ${ }^{34,35}$ Increased RDW may reflect a high oxidative stress level and therefore contribute to poor outcome of TBI patients. Finally, sympathetic-adrenal system and renin-angiotensin system can accelerate erythropoiesis by up-regulating the expression of erythropoietin. ${ }^{36,37}$ After initial mechanical impact, TBI patients often undergo catecholamine surge which is detrimental to multiple organ function. ${ }^{38}$ We have made a reasonable assumption that high RDW is associated with peripheral organ dysfunction and mortality mediated by excessive catecholamine and angiotensin II.

Thrombocytopenia is frequently observed in TBI patients, partly because of blood loss caused by initial open wounds. In addition, platelet count less than $10^{9} / \mathrm{L}$ is one of the diagnostic criteria of coagulopathy, which occurs in $7 \%$ to $63 \%$ of TBI patients. ${ }^{39-41}$ Coagulopathy can lead to increased mortality and high likelihood of progressive hemorrhagic injury in TBI patients. ${ }^{42,43}$ Meanwhile, both platelet dysfunction and low platelet count were found closely correlated with severity of brain injury. ${ }^{44,45}$ Goal-oriented platelet transfusions can improve survival outcome of TBI patients. ${ }^{46}$ In addition to the crucial role in hemostasis and thrombosis, platelets also take part in protective neuroinflammation and improve neuronal plasticity in the damaged neurons area. Serotonin and platelet activating factor released from platelets can modulate neuroinflammation which is beneficial for neuronal rewiring and repair after TBI. ${ }^{47}$

Recent studies have demonstrated that erythrocyte deformability and hematocrit were associated with adenosine $5^{\prime}$-diphosphate-dependent platelet reactivity. ${ }^{48}$ The inherent link between RDW and platelets makes it reasonable to build the novel inflammation marker RPR, which combines the effect of RDW and platelets. The result of our study indicated that RPR was superior to RDW and platelets alone in predicting outcome of TBI patients. One previous study evaluated the prognostic value of RPR 4 days after admission, on TBI patients in United States. ${ }^{29}$ However, many TBI patients may suffer early death within four days after admission, which means RPR on the fourth day may not be used to predict the poor outcome of these patients. Additionally, due to the data derived from a database with limited information, ISS and intracranial radiological signs which are also potential risk factors of mortality in TBI patients, were not recorded. Finally, patients included in this study were mostly elderly, with mean age of 63 . Therefore, we designed our study to overcome these limitations. Our study confirmed that RPR on admission was also effective in predicting outcome of TBI patients in a Chinese medical center. After adjusting for the confounding effects of ISS and intracranial injury types, RPR was still independently associated with mortality of TBI patients. And our constructed model incorporating intracranial injury type and RPR had favorable prognostic value.

There were several limitations in this study. Firstly, this was a single center observational study, so selection bias 
could not be avoided. Secondly, the levels of iron, vitamin B12, and folate which may influence the RDW value, were not measured and recorded. Thirdly, inflammatory markers and neuroendocrine hormone associated with RDW value such as CRP, catecholamine, angiotensin II were not measured.

\section{Conclusion}

RRP is a novel predictive marker which is beneficial for physicians to make treatment strategies for patients with TBI. It is more valuable to evaluate prognosis of TBI patients when utilizing RPR to build a predictive model.

\section{Data Sharing Statement}

The datasets are available from the corresponding author upon reasonable request.

\section{Ethical Approval and Informed Consent}

This study was approved by the Ethics Committee of the West China hospital, Sichuan University and was conducted in accordance with guidelines of the Declaration of Helsinki. Written informed consent of each patient was signed by patients themselves or their legal authorized relatives due to loss of consciousness or akinesia.

\section{Author Contributions}

All authors made a significant contribution to the work reported, whether that is in the conception, study design, execution, acquisition of data, analysis and interpretation, or in all these areas; took part in drafting, revising or critically reviewing the article; gave final approval of the version to be published; have agreed on the journal to which the article has been submitted; and agree to be accountable for all aspects of the work.

\section{Funding}

This study was funded by 1.3.5 project for disciplines of excellence, West China Hospital, Sichuan University (ZYJC18007), Key research and development project of science and technology department of Sichuan Province (2019YFS0392) and Sichuan Science and Technology Program (2021YFS0082 \& 2020YFS0223).

\section{Disclosure}

The authors declare that they have no conflicts of interest.

\section{References}

1. Gardner AJ, Zafonte R. Neuroepidemiology of traumatic brain injury. Handb Clin Neurol. 2016;138:207-223.

2. Dewan MC, Rattani A, Gupta S, et al. Estimating the global incidence of traumatic brain injury. $J$ Neurosurg. 2018;133 (4):1080-1097.

3. Galgano M, Toshkezi G, Qiu X, Russell T, Chin L, Zhao LR. Traumatic brain injury: current treatment strategies and future endeavors. Cell Transplant. 2017;26(7):1118-1130. doi:10.1177/ 0963689717714102

4. Hyder AA, Wunderlich CA, Puvanachandra P, Gururaj G, Kobusingye OC, Neufeld JA. The impact of traumatic brain injuries: a global perspective. NeuroRehabilitation. 2007;22(5):341-353. doi:10.3233/NRE-2007-22502

5. Langlois JA, Rutland-Brown W, Wald MM. The epidemiology and impact of traumatic brain injury: a brief overview. J Head Trauma Rehabil. 2006;21(5):375-378. doi:10.1097/00001199-200609000-00001

6. O'Leary RA, Nichol AD. Pathophysiology of severe traumatic brain injury. J Neurosurg Sci. 2018;62(5):542-548. doi:10.23736/S03905616.18.04501-0

7. Perel P, Arango M, Clayton T, et al. Predicting outcome after traumatic brain injury: practical prognostic models based on large cohort of international patients. BMJ. 2008;336(7641):425-429.

8. Maas AI, Marmarou A, Murray GD, Teasdale SG, Steyerberg EW. Prognosis and clinical trial design in traumatic brain injury: the IMPACT study. J Neurotrauma. 2007;24(2):232-238. doi:10.1089/ neu.2006.0024

9. Han J, King NK, Neilson SJ, Gandhi MP, Ng I. External validation of the CRASH and IMPACT prognostic models in severe traumatic brain injury. $J$ Neurotrauma. 2014;31(13):1146-1152. doi:10.1089/ neu.2013.3003

10. Han F, Liu Y, Cheng S, et al. Diagnosis and survival values of neutrophil-lymphocyte ratio (NLR) and red blood cell distribution width (RDW) in esophageal cancer. Clin Chim Acta. 2019;488:150-158. doi:10.1016/j.cca.2018.10.042

11. Bao Y, Yang M, Jin C, et al. Preoperative hematologic inflammatory markers as prognostic factors in patients with glioma. World Neurosurg. 2018;119:e710-e716. doi:10.1016/j.wneu.2018.07.252

12. Acet H, Ertas F, Akil MA, et al. Relationship between hematologic indices and global registry of acute coronary events risk score in patients with ST-segment elevation myocardial infarction. Clin Appl Thromb Hemost. 2016;22(1):60-68. doi:10.1177/1076029614533145

13. Yayla ME, Ilgen U, Okatan IE, et al. Association of simple hematological parameters with disease manifestations, activity, and severity in patients with systemic sclerosis. Clin Rheumatol. 2020;39 (1):77-83. doi:10.1007/s10067-019-04685-0

14. Taefi A, Huang CC, Kolli K, Ebrahimi S, Patel M. Red cell distribution width to platelet ratio, a useful indicator of liver fibrosis in chronic hepatitis patients. Hepatol Int. 2015;9(3):454-460. doi:10.1007/s12072-015-9638-9

15. Cetinkaya E, Senol K, Saylam B, Tez M. Red cell distribution width to platelet ratio: new and promising prognostic marker in acute pancreatitis. World J Gastroenterol. 2014;20(39):14450-14454. doi:10.3748/wjg.v20.i39.14450

16. Celik T, Balta S, Demir M, et al. Predictive value of admission red cell distribution width-platelet ratio for no-reflow phenomenon in acute ST segment elevation myocardial infarction undergoing primary percutaneous coronary intervention. Cardiol J. 2016;23 (1):84-92. doi:10.5603/CJ.a2015.0070

17. Qiu L, Chen C, Li SJ, et al. Prognostic values of red blood cell distribution width, platelet count, and red cell distribution width-toplatelet ratio for severe burn injury. Sci Rep. 2017;7(1):13720. doi:10.1038/s41598-017-13151-3 
18. Engin H, Cevik G. Prognostic role of RDW-platelet ratio (RPR) in advanced colorectal cancer. Ann Oncol. 2017;28(Suppl 3):iii110. doi:10.1093/annonc/mdx261.313

19. Vreugdenhil G, Lowenberg B, Van Eijk HG, Swaak AJ. Tumor necrosis factor alpha is associated with disease activity and the degree of anemia in patients with rheumatoid arthritis. Eur J Clin Invest. 1992;22(7):488-493. doi:10.1111/j.1365-2362.1992.tb01495.x

20. Vreugdenhil G, Lowenberg B, van Eijk HG, Swaak AJ. Anaemia of chronic disease in rheumatoid arthritis. Raised serum interleukin-6 (IL-6) levels and effects of IL-6 and anti-IL-6 on in vitro erythropoiesis. Rheumatol Int. 1990;10(3):127-130. doi:10.1007/BF02274827

21. Maury CP, Andersson LC, Teppo AM, Partanen S, Juvonen E. Mechanism of anaemia in rheumatoid arthritis: demonstration of raised interleukin 1 beta concentrations in anaemic patients and of interleukin 1 mediated suppression of normal erythropoiesis and proliferation of human erythroleukaemia (HEL) cells in vitro. Ann Rheum Dis. 1988;47(12):972-978. doi:10.1136/ard.47.12.972

22. Gawaz M, Brand K, Dickfeld T, et al. Platelets induce alterations of chemotactic and adhesive properties of endothelial cells mediated through an interleukin-1-dependent mechanism. Implications for atherogenesis. Atherosclerosis. 2000;148(1):75-85. doi:10.1016/ S0021-9150(99)00241-5

23. Tao C, Wang J, Hu X, Ma J, Li H, You C. Clinical value of neutrophil to lymphocyte and platelet to lymphocyte ratio after aneurysmal subarachnoid hemorrhage. Neurocrit Care. 2017;26(3):393-401. doi:10.1007/s12028-016-0332-0

24. Fan Z, Hao L, Chuanyuan T, et al. Neutrophil and platelet to lymphocyte ratios in associating with blood glucose admission predict the functional outcomes of patients with primary brainstem hemorrhage. World Neurosurg. 2018;116:e100-e107. doi:10.1016/j. wneu.2018.04.089

25. Pan H, Fu M, Ge W, Zhou C. The effects of changes in platelet-toneutrophil ratios 24 hours after intravenous thrombolysis on prognosis in acute ischemic stroke patients. Clin Neurol Neurosurg. 2020;190:105739. doi:10.1016/j.clineuro.2020.105739

26. Sadaka F, Doctors N, Pearson T, Snyders B, O’Brien J. Does red cell distribution width predict outcome in traumatic brain injury: comparison to corticosteroid randomization after significant head injury. J Clin Med Res. 2018;10(1):9-12. doi:10.14740/jocmr3173w

27. Bobeff EJ, Fortuniak J, Bryszewski B, et al. Mortality after traumatic brain injury in elderly patients: a new scoring system. World Neurosurg. 2019;128:e129-e147. doi:10.1016/j.wneu.2019.04.060

28. Bae IS, Chun HJ, Yi HJ, Bak KH, Choi KS, Kim DW. Modified glasgow coma scale using serum factors as a prognostic model in traumatic brain injury. World Neurosurg. 2019;126:e959-e964. doi:10.1016/j.wneu.2019.02.197

29. Ge X, Zhu L, Li W, et al. Red cell distribution width to platelet count ratio: a promising routinely available indicator of mortality for acute traumatic brain injury. $J$ Neurotrauma. 2021. doi:10.1089/ neu.2020.7481

30. Lippi G, Targher G, Montagnana M, Salvagno GL, Zoppini G, Guidi GC. Relation between red blood cell distribution width and inflammatory biomarkers in a large cohort of unselected outpatients. Arch Pathol Lab Med. 2009;133(4):628-632. doi:10.5858/133.4.628

31. Lu J, Goh SJ, Tng PY, Deng YY, Ling EA, Moochhala S. Systemic inflammatory response following acute traumatic brain injury. Front Biosci. 2009;14:3795-3813. doi:10.2741/3489

32. Jacome T, Tatum D. Systemic Inflammatory Response Syndrome (SIRS) score independently predicts poor outcome in isolated traumatic brain injury. Neurocrit Care. 2018;28(1):110-116. doi:10.1007/ s12028-017-0410-y
33. Hunziker S, Celi LA, Lee J, Howell MD. Red cell distribution width improves the simplified acute physiology score for risk prediction in unselected critically ill patients. Crit Care. 2012;16(3):R89. doi:10.1186/cc11351

34. Khatri N, Thakur M, Pareek V, Kumar S, Sharma S, Datusalia AK. Oxidative stress: major threat in traumatic brain injury. CNS Neurol Disord Drug Targets. 2018;17(9):689-695. doi:10.2174/ 1871527317666180627120501

35. Rodriguez-Rodriguez A, Egea-Guerrero JJ, Murillo-Cabezas F, CarrilloVico A. Oxidative stress in traumatic brain injury. Curr Med Chem. 2014;21(10):1201-1211. doi:10.2174/0929867321666131217153310

36. Gossmann J, Burkhardt R, Harder S, et al. Angiotensin II infusion increases plasma erythropoietin levels via an angiotensin II type 1 receptor-dependent pathway. Kidney Int. 2001;60(1):83-86. doi:10.1046/j.1523-1755.2001.00773.x

37. Ferrario M, Massa M, Rosti V, et al. Early haemoglobin-independent increase of plasma erythropoietin levels in patients with acute myocardial infarction. Eur Heart J. 2007;28(15):1805-1813. doi:10.1093/ eurheartj/ehm065

38. Zygun D. Non-neurological organ dysfunction in neurocritical care: impact on outcome and etiological considerations. Curr Opin Crit Care. 2005;11(2):139-143. doi:10.1097/01.ccx.0000155356.86241.c0

39. Abdelmalik PA, Boorman DW, Tracy J, Jallo J, Rincon F. Acute traumatic coagulopathy accompanying isolated traumatic brain injury is associated with worse long-term functional and cognitive outcomes. Neurocrit Care. 2016;24(3):361-370. doi:10.1007/s12028-015-0191-0

40. Folkerson LE, Sloan D, Cotton BA, Holcomb JB, Tomasek JS, Wade CE. Predicting progressive hemorrhagic injury from isolated traumatic brain injury and coagulation. Surgery. 2015;158 (3):655-661. doi:10.1016/j.surg.2015.02.029

41. Maegele M, Schochl H, Menovsky T, et al. Coagulopathy and haemorrhagic progression in traumatic brain injury: advances in mechanisms, diagnosis, and management. Lancet Neurol. 2017;16 (8):630-647. doi:10.1016/S1474-4422(17)30197-7

42. Kunio NR, Differding JA, Watson KM, Stucke RS, Schreiber MA. Thrombelastography-identified coagulopathy is associated with increased morbidity and mortality after traumatic brain injury. $\mathrm{Am}$ J Surg. 2012;203(5):584-588. doi:10.1016/j.amjsurg.2011.12.011

43. Allard CB, Scarpelini S, Rhind SG, et al. Abnormal coagulation tests are associated with progression of traumatic intracranial hemorrhage. J Trauma. 2009;67(5):959-967. doi:10.1097/TA.0b013e3181ad5d37

44. Yuan Q, Yu J, Wu X, et al. Prognostic value of coagulation tests for in-hospital mortality in patients with traumatic brain injury. Scand J Trauma Resusc Emerg Med. 2018;26(1):3. doi:10.1186/s13049-0170471-0

45. Daley MJ, Enright Z, Nguyen J, et al. Adenosine diphosphate platelet dysfunction on thromboelastogram is independently associated with increased morality in traumatic brain injury. Eur J Trauma Emerg Surg. 2017;43(1):105-111. doi:10.1007/s00068-016-0643-z

46. Furay E, Daley M, Teixeira PG, et al. Goal-directed platelet transfusions correct platelet dysfunction and may improve survival in patients with severe traumatic brain injury. J Trauma Acute Care Surg. 2018;85(5):881-887. doi:10.1097/TA.0000000000002047

47. Dukhinova M, Kuznetsova I, Kopeikina E, et al. Platelets mediate protective neuroinflammation and promote neuronal plasticity at the site of neuronal injury. Brain Behav Immun. 2018;74:7-27. doi:10.1016/j.bbi.2018.09.009

48. Cecchi E, Marcucci R, Paniccia R, et al. Effect of blood hematocrit and erythrocyte deformability on adenosine 5 '-diphosphate platelet reactivity in patients with acute coronary syndromes on dual antiplatelet therapy. Am J Cardiol. 2009;104(6):764-768. doi:10.1016/j. amjcard.2009.05.005 


\section{Publish your work in this journal}

Therapeutics and Clinical Risk Management is an international, peerreviewed journal of clinical therapeutics and risk management, focusing on concise rapid reporting of clinical studies in all therapeutic areas, outcomes, safety, and programs for the effective, safe, and sustained use of medicines. This journal is indexed on PubMed Central, CAS,
EMBase, Scopus and the Elsevier Bibliographic databases. The manuscript management system is completely online and includes a very quick and fair peer-review system, which is all easy to use. Visit http://www.dovepress.com/testimonials.php to read real quotes from published authors. 